\title{
Atividades desenvolvidas pela equipe de enfermagem no contexto do HIV/AIDS no
}

\section{Brasil}

\author{
Activities performed by the nursing staff in the context of HIV/AIDS in Brazil \\ Actividades realizadas por el personal de enfermería en el contexto del VIH/SIDA en Brasil
}

Recebido: 03/11/2021 | Revisado: 12/11/2021 | Aceito: 21/11/2021 | Publicado: 01/12/2021

Denize Cristina de Oliveira ORCID: https://orcid.org/0000-0002-0830-0935 Universidade do Estado do Rio de Janeiro, Brasil

E-mail: dcouerj@gmail.com

Sergio Corrêa Marques

ORCID: https://orcid.org/0000-0003-2597-4875 Universidade do Estado do Rio de Janeiro, Brasil

E-mail: sergiocmarques@uol.com.br

Thelma Spindola

ORCID: https://orcid.org/0000-0002-1785-5828 Universidade do Estado do Rio de Janeiro, Brasil

E-mail: tspindola.uerj@gmail.com

Elisabeth Teixeira

ORCID: https://orcid.org/0000-0002-5401-8105 Universidade do Estado do Pará, Brasi

E-mail: etfelipe@ hotmail.com

Hellen Pollyanna Mantelo Cecilio

ORCID: https://orcid.org/0000-0002-6597-432X

Universidade Federal de Mato Grosso do Sul, Brasil

E-mail: pollymantelo@gmail.com

Yndira Yta Machado

ORCID: https://orcid.org/0000-0003-0692-7299

Universidade do Estado do Rio de Janeiro, Brasil

E-mail: yndiramachado@gmail.com

Juliana Pereira Domingues

ORCID: https://orcid.org/0000-0002-0966-4992

Universidade do Estado do Rio de Janeiro, Brasil

E-mail: pdominguesjuliana@gmail.com

Renata Lacerda Marques Stefaisk

ORCID: https://orcid.org/ 0000-0001-5592-7565

Universidade do Estado do Rio de Janeiro

E-mail: renata_350@hotmail.com

Camila Laporte Almeida de Souza

ORCID: https://orcid.org/0000-0002-3845-9226

Universidade do Estado do Rio de Janeiro, Brasil

E-mail: camila.laporte@hotmail.com

Henrique Franscisco de Sena

ORCID: https://orcid.org/0000-0003-0146-298X

Universidade do Estado do Rio de Janeiro, Brasil

E-mail: henrique.francisco.sena@hotmail.com

Keila Pereira da Silva

ORCID: https://orcid.org/0000-0002-2753-9685

Universidade do Estado do Rio de Janeiro, Brasil

E-mail: keilaps1997@gmail.com

Suzana da Silva Castro

ORCID: https://orcid.org/0000-0001-7347-0853

Universidade do Estado do Rio de Janeiro, Brasil

E-mail: castro.susi@hotmail.com

\begin{abstract}
Resumo
Objetivo: Descrever as ações desenvolvidas pelos profissionais de enfermagem nos serviços de atendimento às pessoas vivendo com HIV. Metodologia: Estudo de campo transversal, quantitativo, baseado nas atribuições da equipe de enfermagem no Programa de Atenção ao HIV/aids do Brasil. Foram estudadas 12 cidades nas cinco regiões geográficas brasileiras. Realizou-se a coleta de dados com 54 gestores de unidades de saúde, por meio de questionário estandardizado. As variáveis analisadas foram as atividades e os profissionais responsáveis pela realização das ações de atenção às pessoas vivendo com HIV. Resultados: Das 54 instituições estudadas, 18,5\% estão na região norte,
\end{abstract}


$16,7 \%$ nordeste, $14,8 \%$ centro-oeste, $46,3 \%$ sudeste e $3,7 \%$ sul. Entre as atividades atribuídas à equipe de enfermagem, os enfermeiros foram mencionados como responsáveis pelas atividades de aconselhamento individual e coletivo (77,9 e 73,4\%), oferta de exame anti-HIV (74,5\%), consulta de enfermagem (95,1\%), primeira consulta ao cliente com HIV $(88,5 \%)$ e atendimento domiciliar $(84,6 \%)$. Observou-se que os atendimentos psicológico e social foram apontados como atividade própria dos enfermeiros, apesar de serem da competência de outros profissionais; além de ações exclusivas do enfermeiro sendo atribuídas aos técnicos e auxiliares de enfermagem, como a consulta de enfermagem. Conclusão: A maior parte das ações realizadas nos serviços foi associada ao enfermeiro. Essa associação demonstra o reconhecimento da importância desse profissional pelos gestores, mas também pouca clareza sobre as atribuições da equipe de enfermagem revelando uma fragilidade gerencial das equipes de saúde.

Palavras-chave: Serviços de enfermagem; HIV; Serviços de saúde; Saúde pública; Enfermagem.

\begin{abstract}
Objective: To describe the actions taken by nursing professionals in the care services of people living with HIV. Methodology: Cross-sectional field study, quantitative, based on the attributions of the nursing team in the HIV/AIDS Care Program of Brazil. Twelve Brazilian cities distributed in five brazilians geographic regions were studied. Data were collected with 54 managers from health units through a standardized questionnaire. The variables analyzed was activities and professionals responsible for carrying out care actions for people living with HIV. Results: Of the 54 institutions studied, $18.5 \%$ are in the North region, $16.7 \%$ in the Northeast, $14.8 \%$ in the Midwest, $46.3 \%$ in the Southeast and $3.7 \%$ in the South. Among the activities assigned to the nursing team, nurses were mentioned as responsible for individual and collective counseling activities (77.9 and 73.4\%), HIV testing offer (74.5\%), nursing consultation $(95.1 \%)$, first consultation with clients with HIV $(88.5 \%)$ and home care $(84.6 \%)$. The performance of psychological and social care was pointed out as the nurses' own activity, despite being the competence of other professionals; in addition to exclusive nurses' actions being assigned to nursing technicians and assistants, such as nursing consultations. Conclusion: Most of the actions performed in the services were associated with nurses. This association demonstrates the recognition of the importance of this professional by the managers, but also little clarity about the attributions of the nursing team revealing a managerial weakness of health teams.
\end{abstract}

Keywords: Nursing services; HIV; Health services; Public health; Nursing.

\title{
Resumen
}

Objetivo: Describir las acciones realizadas por los profesionales de enfermería en los servicios de atención de personas que viven con VIH. Metodología: Estudio de campo transversal cuantitativo, basado en las atribuciones del equipo de enfermería en el Programa de Atención al SIDA en Brasil. Se estudiaron 12 ciudades en las cinco regiones geográficas brasileñas. La recolección de datos se realizo con 54 gerentes de unidades de salud a través de un cuestionario estandarizado. Las variables analizadas incluyen las actividades y los profesionales responsables por las acciones de atención a las personas que viven con VIH. Resultados: De las 54 instituciones estudiadas, el 46,3\% se encuentran en la region Sureste, el 18,5\% en el Norte, el 16,7\% en el Nordeste, el 14,8\% en el Medio Oeste y el 3,7\% en el Sur. Entre las actividades asignadas al equipo de enfermería se mencionaron enfermeras como responsables de las actividades de consulta de enfermería (95,1\%), primera consulta con clientes con VIH $(88,5 \%)$, atención domiciliaria (84,6\%), consejería individual y colectiva $(77,9$ y 73,4\%), pruebas anti-VIH (74,5\%). La realización de la atención psicológica y social se identificó como actividad propia del enfermero, a pesar de ser responsabilidad de otros profesionales; además de la asignación de acciones exclusivas de enfermeras a técnicos y auxiliares de enfermería, como consultas de enfermería. Conclusión: La mayoría de las acciones realizadas en los servicios estuvieron asociadas al enfermero. Esta asociación demuestra el reconocimiento de la importancia de este profesional por parte de los directivos, pero también poca claridad sobre las atribuciones del equipo de enfermería revelando una debilidad gerencial de los equipos de salud.

Palabras clave: Servicios de enfermeira; VIH; Servicios de salud; Salud pública; Enfermería.

\section{Introdução}

A importância da aids no cenário nacional e internacional é indiscutível, tanto por sua expressão epidemiológica, quanto para a organização dos serviços de saúde. No período de 2007 até junho de 2020 foram notificados no Sistema de Informação de Agravos de Notificação (Sinan), 342.459 casos de infecção pelo Vírus da Imunodeficiência Humana (HIV) em todo território nacional (Brasil, 2020; Brasil, 2017a).

A estruturação, em âmbito nacional, dos serviços de saúde para enfrentar a epidemia da Síndrome da Imunodeficiência Humana (aids) teve início em 1988 de uma série de programas direcionados à prevenção e atenção a portadores de HIV/aids e outras IST (Infecções Sexualmente Transmissíveis). Em 1999 foi criado o Programa Nacional de DST/Aids (PN DST/aids), sob responsabilidade do Ministério da Saúde (MS), como produto dessa série de programas surgidos 
ao longo dos anos desde a descoberta da aids. O PN DST/aids visa reduzir a incidência de HIV/aids e outras IST e melhorar a qualidade de vida das pessoas portadoras destas doenças. Para tanto, foram definidas diretrizes que englobam o aumento da cobertura das ações preventivas, de diagnóstico e de tratamento; a melhoria da qualidade dos serviços públicos oferecidos, a redução da transmissão vertical de sífilis e HIV, bem como a redução da discriminação aos portadores. Como parte dessas diretrizes, em 1996 o Brasil foi pioneiro no acesso universal e gratuito à terapia antirretroviral (TARV), no Sistema Único de Saúde (SUS) (Brasil, 1999a, b).

O PN DST/aids foi estruturado a partir dos seguintes serviços: Centro de Testagem e Aconselhamento (CTA); Serviço de Assistência Especializada (SAE); Centro de Referência e Treinamento (CRT); Assistência Domiciliar Terapêutica em Aids e Unidade Dispensadora de Medicamentos (Brasil, 2013). O SAE caracteriza-se por ser uma unidade ambulatorial, voltada à atenção integral às pessoas vivendo com HIV (PVHIV), que tem o objetivo de prestar atendimento integral e de qualidade aos usuários por meio de uma equipe de saúde multiprofissional composta, minimamente, por médicos, enfermeiros, auxiliares ou técnicos de enfermagem, assistente social e/ou psicólogo. Os CTAs são serviços de saúde que realizam ações de diagnóstico e prevenção de ISTs, realizando testes para HIV, sífilis e hepatites B e C, de acordo com as normas definidas pelo Ministério da Saúde (Brasil, 2013).

Diante da evolução da epidemia de aids, com perfis distintos nos diferentes centros urbanos e nas diversas regiões do Brasil, as práticas profissionais de saúde necessitaram ser transformadas em função dos contextos das políticas de saúde, dos elementos institucionais e éticos, das práticas e dos valores sociais. Dentre as profissões da saúde que atuam no cuidado às PVHIV, a equipe de enfermagem desenvolve suas ações no sentido da ampliação da noção de cuidado em saúde e da sua prática, orientando suas ações por um direcionamento multidimensional do fenômeno de viver com HIV, que envolve questões psicológicas, espirituais e emocionais, além daquelas mais diretamente afeitas a doença física, sendo o cuidado ampliado um grande desafio para a qualificação da atenção às PVHIV (Oliveira et al., 2009).

Este estudo justifica-se pelo fato de a equipe de enfermagem ser fundamental no desenvolvimento da linha de cuidado de saúde das PVHIV, sendo aquela responsável pelo maior número de ações no PN DST/aids visto que está presente em todas as etapas do tratamento do HIV desde o diagnóstico. Assim, a presente pesquisa é relevante para o reconhecimento da atuação da equipe de enfermagem nesse cenário, além do reconhecimento da visibilidade gerencial dessa atuação.

Neste sentido, considerando a invisibilidade histórica do trabalho de enfermagem, delimitou-se como objetivo descrever as ações desenvolvidas pelos profissionais da equipe de enfermagem nos serviços de atendimento à PVHIV no Brasil.

\section{Metodologia}

Trata-se de uma pesquisa de campo, transversal, com abordagem quantitativa, fundamentado nas atribuições da equipe de enfermagem no PN DST/aids do Brasil. A pesquisa faz parte do projeto multicêntrico nacional intitulado "As transformações do cuidado em saúde em tempos de aids: memórias e representações de profissionais de saúde e enfermagem no Brasil".

Foram estudados 54 gestores de serviços de saúde nos quais era desenvolvido o PN DST/aids, em 12 municípios, contemplando as cinco regiões brasileiras. O quantitativo de serviços de saúde envolvidas foram: Rio de Janeiro (19), Niterói (05), Rio Grande (02), Cuiabá (08), Jequié (01), Vitória da Conquista (01), Salvador (01), Recife (07), Manaus (05), Coari (01), Santarém (01) e Belém (03). A definição amostral considerou a ponderação das diferentes cidades e regiões do Brasil em termos de número de sujeitos atendidos e as exigências mínimas para comparação dos dados. Os critérios de inclusão foram: unidades de Atenção Primária à Saúde e ambulatórios hospitalares que desenvolviam atendimento às PVHIV em diferentes regiões do Brasil. O quantitativo de participantes foi definido por amostra intencional, definida a partir do quantitativo total de 
gestores dos serviços participantes do estudo.

A coleta de dados foi finalizada em 2016, por pesquisadores integrantes do projeto supracitado nos respectivos municípios. Foi utilizado um questionário estandardizado composto por variáveis relativas à caracterização dos serviços, às atividades e aos profissionais responsáveis pela realização das ações de atenção à PVHIV nos serviços estudados. Os dados coletados foram digitados em planilha eletrônica do Microsoft Excel® 2010 e, posteriormente, transpostos para o programa estatístico Software Statistical Package for the Social Sciences (SPSS)®, versão 20. Foi realizada análise descritiva dos dados por meio de frequências absolutas e relativas.

O projeto foi aprovado pelo CONEP - registro 16755, por meio do parecer 053 e, posteriormente, pelos Comitês de Ética dos municípios integrantes da pesquisa. Para a realização da coleta de dados foi encaminhada uma solicitação às diretorias dos serviços de saúde, anexando-se uma cópia do projeto de pesquisa e a carta de aprovação do Comitê de Ética. Além disso, os participantes assinaram o Termo de Consentimento Livre e Esclarecido.

\section{Resultados}

A distribuição geográfica das 54 instituições estudadas revelou que 46,3\% (25) se encontravam na região sudeste, $18,5 \%$ (10) na região norte, $16,7 \%$ (9) na região nordeste, $14,8 \%$ (8) na região centro-oeste e 3,7\% (2) na região sul. Esses serviços caracterizavam-se como: 26,4\% ambulatório vinculado a hospital; 22,7\% unidade básica de saúde, 22,7\% ambulatório de assistência de várias especialidades, não vinculado a hospital; 16,9\% ambulatório de assistência especializada às PVHIV, não vinculado ao hospital; e, 11,3\% como unidade hospitalar.

Os dados da distribuição das instituições gestoras e dos tipos de serviço estão apresentados na Tabela 1.

Tabela 1. Tipos de instituições e de serviços de assistência às pessoas vivendo com HIV no Brasil, 2021.

\begin{tabular}{lccc}
\cline { 1 - 2 } Instituição gestora e tipo de serviço & f & \% \\
\cline { 1 - 2 } Instituição gestora & 36 & 66,6 \\
Secretaria Municipal de Saúde & 10 & 18,5 \\
Universidade Pública & 6 & 11,1 \\
Secretaria Estatual de Saúde & 1 & 1,9 \\
Universidade Privada & 1 & 1,9 \\
Sem resposta & & \\
Tipo de serviço & 20 & 37,1 \\
Centro de Testagem e Aconselhamento (CTA) & 16 & 29,6 \\
Serviço de Assistência Especializada (SAE) & 16 & 29,6 \\
Ambulatório de Assistência Especializada às & & \\
pessoas com HIV/aids & 2 & 3,7 \\
Centro de Referência HIV/aids & $\mathbf{5 4}$ & $\mathbf{1 0 0}$ \\
\hline Total & &
\end{tabular}

Fonte: Autores.

Quanto à rotina de dias e horários de atendimento à PVHIV, observou-se que em 75,9\% dos serviços o ambulatório estava aberto ao público durante cinco dias úteis da semana; em 9,3\% em quatro dias por semana; em 5,6\% em dois e três dias por semana e em 3,7\% em mais de cinco dias. No que se refere ao atendimento diário, 53,7\% funcionavam oito horas diárias; $24,1 \%$ funcionavam 12 horas por dia, enquanto $11,1 \%$ e $7,4 \%$ funcionavam seis e quatro horas diárias, respectivamente, apenas $3,7 \%$ dos serviços funcionavam mais de 12 horas por dia. 
A maioria dos serviços estava localizada em regiões de grande disponibilidade de transporte coletivo $(90,7 \%)$. Na percepção dos gestores, 51,9\% dos prédios possuíam planta física que facilitava a circulação interna; 77,8\% referiram que o prédio não apresentava barreira física de acesso, no entanto, em 20,4\% dos prédios essas barreiras físicas foram apontadas pelos gestores, tais como escadas e outros que dificultavam a circulação interna e o acesso as atividades.

A análise da distribuição do quantitativo de profissionais constituintes da equipe de enfermagem, disponíveis para atendimento à pessoa que vive com HIV nos serviços, encontra-se descrito na Tabela 2.

Tabela 2. Distribuição dos profissionais da equipe de enfermagem em função do seu quantitativo nos serviços de atenção às pessoas que vivem com HIV no Brasil, 2021.

\begin{tabular}{lcccccc}
\hline $\begin{array}{l}\text { Quantitativo de } \\
\text { profissionais }\end{array}$ & Enfermeiro & \multicolumn{2}{c}{ Técnico de } & \multicolumn{2}{c}{$\begin{array}{c}\text { Auxiliar de } \\
\text { Enfermagem }\end{array}$} & \multicolumn{2}{c}{ Enfermagem } \\
& f & \% & f & \% & f & \% \\
\hline 0 & 8 & 14,8 & 17 & 31,5 & 40 & 74,1 \\
1 & 23 & 42,6 & 8 & 14,8 & 4 & 7,4 \\
2 ou 3 & 15 & 27,8 & 14 & 25,9 & 7 & 12,9 \\
4 ou 5 & 4 & 7,4 & 10 & 18,5 & 2 & 3,7 \\
6 ou mais & 4 & 7,4 & 5 & 9,3 & 1 & 1,9 \\
\hline Total de serviços & $\mathbf{5 4}$ & $\mathbf{1 0 0}$ & $\mathbf{5 4}$ & $\mathbf{1 0 0}$ & $\mathbf{5 4}$ & $\mathbf{1 0 0}$ \\
\hline & \multicolumn{2}{l}{}
\end{tabular}

A distribuição dos quantitativos de profissionais participantes das equipes de enfermagem aponta como principal resultado a ausência de Enfermeiros em 14,8\% dos serviços, além da ausência de Técnico de Enfermagem em 31,5\% e de Auxiliares de Enfermagem em 74,1\% das unidades analisadas. Destaca-se, ainda, a maior concentração de profissionais entre os Técnico de Enfermagem com 53,7\% com mais de dois profissionais por serviço; e de 42,6\% com um Enfermeiro por serviço.

Em relação às atividades preconizadas pelo Ministério da Saúde para o PN DST/aids, a Tabela 3 apresenta aquelas atribuídas à equipe de enfermagem pelos gestores dos serviços de saúde.

Tabela 3. Atividades realizadas pela equipe de enfermagem nos serviços de assistência às pessoas que vivem com HIV no Brasil, 2021.

\begin{tabular}{|c|c|c|c|c|c|c|c|c|}
\hline \multirow[t]{2}{*}{ Atividades/Ações } & \multicolumn{2}{|c|}{ Enfermeiro } & \multicolumn{2}{|c|}{ Técnico } & \multicolumn{2}{|c|}{ Auxiliar } & \multicolumn{2}{|c|}{ Total ações } \\
\hline & f & $\%$ & f & $\%$ & f & $\%$ & f & $\%$ \\
\hline Aconselhamento individual & 109 & 77,9 & 26 & 18,6 & 5 & 3,5 & 140 & 100 \\
\hline Aconselhamento coletivo & 69 & 73,4 & 18 & 19,1 & 7 & 7,5 & 94 & 100 \\
\hline Atendimento pré-consulta & 21 & 42,9 & 19 & 38,8 & 9 & 18,3 & 49 & 100 \\
\hline Oferta de exame anti-HIV & 35 & 74,5 & 9 & 19,1 & 3 & 6,4 & 47 & 100 \\
\hline $\begin{array}{l}\text { Coleta de material para } \\
\text { exame anti-HIV }\end{array}$ & 14 & 33,3 & 22 & 52,4 & 6 & 14,3 & 42 & 100 \\
\hline Consulta de enfermagem & 39 & 95,1 & 2 & 4,9 & - & - & 41 & 100 \\
\hline Atendimento pós-consulta & 20 & 57,2 & 11 & 31,4 & 4 & 11,4 & 35 & 100 \\
\hline $\begin{array}{l}\text { Primeira consulta ao cliente } \\
\text { com HIV }\end{array}$ & 23 & 88,5 & 3 & 11,5 & - & - & 26 & 100 \\
\hline $\begin{array}{l}\text { Dispensação de } \\
\text { medicamentos ARV }\end{array}$ & 8 & 50 & 8 & 50 & - & - & 16 & 100 \\
\hline Atendimento domiciliar & 11 & 84,6 & 1 & 7,7 & 1 & 7,7 & 13 & 100 \\
\hline $\begin{array}{l}\text { Atendimento } \\
\text { psicológico/social }\end{array}$ & 7 & 100 & - & - & - & - & 7 & 100 \\
\hline Total de ações & 356 & 69,8 & 119 & 23,3 & 35 & 6,9 & 510 & 100 \\
\hline
\end{tabular}

Fonte: Autores. 
Conforme a Tabela 3, dentre as atividades referidas pelos gestores como sendo realizadas pela equipe de enfermagem nos serviços de atendimento à PVHIV, ressalta-se que a maior parte delas foi apontada como sendo realizada pelos enfermeiros $(69,8 \%)$ e apenas $30,2 \%$ pelos técnicos e auxiliares de enfermagem. A distribuição das atividades apontadas como sendo desenvolvidas pelos enfermeiros em relação ao total das atividades da equipe são, em ordem decrescente: atendimento psicológico/social (100\%), consulta de enfermagem $(95,1 \%)$, primeira consulta ao cliente com HIV $(88,5 \%)$, atendimento domiciliar (84,6\%), aconselhamento individual (77,9\%), oferta de exame anti-HIV (74,5\%), aconselhamento coletivo (73,4\%), atendimento pós-consulta (57,2\%), dispensação de medicamentos ARV (50\%), atendimento pré-consulta (42,9\%) e coleta de material para exame anti-HIV (33,3\%). Os enfermeiros são mencionados como responsáveis por atividades educativas desenvolvidas em 77,9\% do total das ações, o que expressa a invisibilidade das atividades educativas desenvolvidas pelos auxiliares e técnicos de enfermagem.

Outros resultados apontam a baixa frequência de atribuição pelos gestores de ações exclusivas ou preferenciais da equipe de enfermagem, como aconselhamento individual, aconselhamento coletivo, atendimento pré-consulta, atendimento pós-consulta, atendimento domiciliar, coleta de material para exame anti-HIV e consulta de enfermagem, essenciais para o acompanhamento das PVHIV e de execução obrigatória no seu acompanhamento nos serviços de saúde.

Foram observados alguns vieses de atribuição de funções pelos gestores, especialmente em referência a realização da consulta de enfermagem por profissionais de nível técnico (4,9\%), além da primeira consulta à pessoa com HIV (11,5\%) e atendimentos psicológicos, sociais e farmacêuticos desenvolvidos pela equipe de enfermagem.

\section{Discussão}

Os serviços de saúde incluídos neste estudo estavam situados, majoritariamente, na região sudeste, principalmente no município do Rio de Janeiro, considerando sua importância no cenário do surgimento e desenvolvimento da aids no Brasil, bem como a característica centralizada da rede de atenção nas regiões mais ricas do Brasil (Oliveira et al., 2009). Os dados encontrados sinalizam que os serviços eram desenvolvidos em ambulatórios vinculados a hospitais, ambulatórios de assistência a várias especialidades e em centros municipais de saúde, diferente dos resultados encontrados na avaliação da qualidade dos serviços ambulatoriais do SUS que assistem adultos vivendo com HIV no Brasil, em que se observou que 40,6\% dos serviços estavam localizados em ambulatórios de assistência a várias especialidades (Nemes, 2011). Destaca-se que a descentralização das ações de atenção às pessoas que vivem com HIV para a Estratégia de Saúde da Família estava se iniciando e havia se efetivado apenas parcialmente em nível nacional, no momento da coleta de dados desta pesquisa, o que certamente determinou a mudança desse perfil nos anos posteriores.

A instituição gestora da maioria dos serviços era a Secretaria Municipal de Saúde, assim como na pesquisa Qualiaids (Nemes, 2011) e em consonância com as diretrizes do SUS. Na consolidação das diversas leis, programas e coordenações de saúde, a implantação do SAE e CTA foi delegada às coordenações estaduais ou municipais, com vistas à descentralização, que se apresentava como desafio a enfrentar no momento da coleta de dados (Villarinho et al., 2013).

Os serviços de assistência, em sua maioria, foram caracterizados como CTA, seguidos do SAE. A política nacional recomenda que todo o processo de detecção do HIV nos serviços deve ser realizado no CTA, de forma sigilosa, oferecendo a quem realiza o rastreamento a possibilidade de ser acompanhado por uma equipe de profissionais de saúde que orientará sobre o resultado final do exame, independente do mesmo ser positivo ou negativo (Brasil, 2013). Quando o resultado é positivo, os CTA são responsáveis por encaminhar o cliente para tratamento nos serviços de referência ou SAE.

Os serviços oferecidos pelos CTA devem seguir os princípios de acessibilidade e gratuidade, estando situados em lugares de fácil acesso para a população, baseados no anonimato. (Villarinho et al., 2013; Brasil, 2017a). Dentre as atividades que devem ser desenvolvidas pelo SAE estão os cuidados de enfermagem, orientação e apoio psicológico, atendimento médico 
e odontológico, controle e distribuição de antirretrovirais, distribuição de preservativos, atividades educativas para adesão à terapia antirretroviral e para prevenção e controle de IST e aids, dentre outras. Deste modo, o SAE consiste em um cenário importante de compartilhamento de saberes e experiências entre os diversos atores sociais implicados na dinâmica do enfrentamento do HIV e da aids e da configuração de representações e práticas em relação à aids e à qualidade de vida das PVHIV (Brasil, 2013; Brasil, 2008a).

Apesar da descentralização da atenção a esse grupo a partir da gestão compartilhada entre a unidade básica de saúde e o SAE ter sido recomendada pelo MS (Brasil, 2017b) como parte da política de atenção para reorganização dos fluxos de atendimento nos anos seguintes à coleta de dados, esse processo estava em etapa de implantação em apenas parte dos serviços estudados, mantendo-se a organização preconizada pelo PN DST/aids de centralização do acompanhamento no SAE. Observou-se que os SAEs e ambulatórios de especialidades continuavam a representar os principais responsáveis pelas ações de acompanhamento desse grupo. Nesse aspecto, deve-se destacar que a aids coloca-se entre as doenças com caráter estigmatizante, o que leva frequentemente os sujeitos que necessitam de atenção e acompanhamento a optarem por serviços distantes da residência e que assegurem o anonimato, inclusive no acesso.

Com relação a rotina de atendimento dos serviços, a maioria funcionava em cinco ou mais dias por semana e de oito a 12 ou mais horas diárias. Estes dados corroboram a pesquisa Qualiaids em que 85,6\% dos serviços funcionam mais de cinco dias por semana e $81,6 \%$, oito ou mais horas diárias. Ainda de acordo com esse estudo, a maioria dos serviços estava localizada em regiões de fácil disponibilidade de transporte coletivo. Porém, apesar da maioria dos gestores indicarem que os prédios possuíam planta física adequada, um número importante de serviços referiu ter barreiras físicas que dificultavam a circulação interna, conforme também observado no estudo de Nemes (2011).

Em relação a existência e concentração de profissionais de enfermagem nos serviços, a maioria possuía, pelo menos, um enfermeiro, o que corrobora com os resultados da pesquisa Qualiaids que indicou que em $91 \%$ dos serviços havia pelo menos um enfermeiro na equipe e em 88,8\% pelo menos um auxiliar ou técnico de enfermagem (Nemes, 2011). No entanto, também se observou a ausência do Enfermeiro em alguns serviços analisados, mesmo considerando a exigência de ações específicas desse profissional nos serviços de atenção à pessoa com HIV, o que aponta uma fragilidade da composição das equipes de atendimento. Destaca-se, ainda, a ausência de Auxiliares de Enfermagem na maior parte dos serviços e a maior concentração de Técnicos de Enfermagem nos serviços analisados, podendo apontar o processo de substituição de Auxiliares por Técnicos de Enfermagem já em etapa avançada nesses serviços.

Em estudo realizado em três SAE de Recife-PE identificou-se que todos os serviços dispunham de enfermeiros na equipe, em um SAE havia três enfermeiros e nos outros dois, um enfermeiro em cada; em relação aos técnicos de enfermagem, em um serviço havia três técnicos na equipe, em outro, dois técnicos e um não possuía esse profissional na equipe. Os três SAE incluídos no estudo em Recife não contavam com auxiliares de enfermagem na equipe (Abrão et al., 2014).

Os profissionais de saúde têm papel fundamental na atuação com PVHIV, necessitando de capacitações constantes, valorização profissional e estímulo ao trabalho articulado e em equipe. Destaca-se que os profissionais de saúde são responsáveis por prestar assistência integral, de qualidade e livre de preconceitos, devem garantir a todos as pessoas o direito de serem tratados com respeito, dignidade, igualdade e justiça, obedecendo assim a um dos princípios do SUS, que é a equidade (Abrão et al., 2014). Para o alcance desse objetivo, a equipe de enfermagem é essencial, articulando-se aos demais membros da equipe de saúde para o cuidado humanizado e integral.

As atividades atribuídas pelos gestores à equipe de enfermagem abarcam atividades finalísticas e atividades meio. As atividades finalísticas são atendimento psicológico/social, consulta de enfermagem, primeira consulta ao cliente com HIV, atendimento domiciliar, aconselhamento individual e coletivo, dispensação de medicamentos ARV. As atividades meio são atendimento pré e pós-consulta, oferta de exame anti-HIV e coleta de material para exame anti-HIV, que se concretizam em 
estreita relação de dependência a outras atividades, como consulta médica, consulta de enfermagem e outras.

$\mathrm{Na}$ análise das ações desenvolvidas pela equipe de enfermagem foi apontado pelos gestores que a maior parte delas era realizada pelos enfermeiros, o que sugere pouca clareza quanto a especificidade do trabalho dos diferentes profissionais nessa equipe. Algumas atividades de competência exclusiva do Enfermeiro foram apontadas, como a consulta de enfermagem e a primeira consulta ao cliente com HIV, assim como outras compartilhadas pela equipe, como atendimento domiciliar, aconselhamento individual, oferta de exame anti-HIV e aconselhamento coletivo. Além destas, a realização dos atendimentos psicológico e social foi apontada como atividade própria dos enfermeiros, apesar de serem da competência de outros profissionais.

O papel do enfermeiro e a compreensão da sua importância no cenário do SUS mostram as potencialidades dessa profissão (Pereira, Fonseca, Oliveira, Fernandes \& Marques, 2015). Desse modo, as ações de enfermagem visam a promoção de estratégias de acolhimento à PVHIV; a escuta ativa, através da consideração das singularidades de cada um, direcionando o cuidado; e o estímulo ao apoio familiar como contribuição na adesão ao tratamento (Ribeiro et al, 2021).

As atividades preconizadas para as equipes multiprofissionais dos serviços que atendem PVHIV são embasadas nos cuidados integrais, nos cuidados de enfermagem, na orientação e no apoio psicológico, nos atendimentos médico e odontológico, no controle e distribuição de antirretrovirais, orientação farmacêutica, realização de exames de monitoramento, distribuição de insumos de prevenção, atividades educativas para adesão ao tratamento e para prevenção e controle de IST e aids (Brasil, 2020). Quanto às ações de cuidado recomendadas para a equipe de enfermagem, estas são compostas por uma grande diversidade de atividades técnicas que estão presentes na maioria dos momentos do trabalho assistencial. Os espaços de atendimento de enfermagem criam oportunidades de diálogo ampliado, pelo fato de não serem apenas centrados nas questões clínicas. Sendo assim, cabe ao enfermeiro realizar a supervisão da equipe de enfermagem, a consulta de enfermagem, a visita domiciliar, o acolhimento, os grupos de adesão, a notificação epidemiológica, pré e pós consulta médica para os casos prioritários, atividades educativas e de controle de faltosos (Brasil, 2008b; 2018).

De acordo com o Ministério da Saúde, ao procurar um CTA o usuário deve ser acolhido e participar de uma atividade de aconselhamento. $\mathrm{O}$ aconselhamento é entendido como um processo de escuta ativa, individualizado e centrado no cliente, com vistas ao estabelecimento de uma relação de confiança entre o profissional e a PVHIV. A prática de aconselhamento às PVHIV envolve a necessidade de o indivíduo receber suporte emocional adequado para lidar com sua nova condição e participar ativamente do seu processo terapêutico (Ministério da Saúde, 2020). O que se observou neste estudo foi que essa prática é identificada pelos gestores como atividade própria do enfermeiro e não da equipe de enfermagem. Esse é um viés na concepção do que se configura como aconselhamento, o que deve propiciar a reflexão sobre os valores, atitudes e comportamentos adotados nas ações de atenção ao grupo. Tal atividade deveria ser uma prática compartilhada por toda a equipe de saúde e de enfermagem, inclusive técnicos e auxiliares, devidamente capacitados.

Além do aconselhamento, outras ações de prevenção devem ser realizadas pelos serviços de saúde, incluindo a disponibilização de insumos de prevenção para a população geral, tais como preservativos masculinos e femininos, gel lubrificante para profissionais do sexo e homens que fazem sexo com homens e kits de redução de danos para pessoas que usam drogas (Brasil, 2013). Essas ações não foram observadas neste estudo.

Em relação às atividades de educação em saúde, a equipe de enfermagem desenvolve atividades individuais e coletivas e são, na sua grande maioria, atribuídas pelos gestores ao enfermeiro. Esses resultados também foram encontrados em uma pesquisa que indicou que a configuração do agir educativo no contexto do HIV/aids se dá como ato-tarefa de orientaçãoinformação para a adoção de modos de vida saudáveis e práticas seguras. Ainda, a educação em saúde pode se desdobrar em diferentes tipologias e modelos, além de anunciar (ou não) as transformações e transições, revelando nuances e interfaces de construções sociocognitivas distintas. Para os profissionais de enfermagem, a educação em saúde coloca-se como ação 
destacada no âmbito do cuidado de enfermagem, isto é, ao considerar-se que a humanização do cuidado se faz presente em ações resolutivas, avaliação clínica e reconhecimento do direito da pessoa à informação, há uma associação entre educação em saúde e cuidado humanizado (Teixeira \& Oliveira, 2014).

As práticas educativas, inseridas no contexto específico das unidades de referência para PVHIV, acontecem nas ocasiões dos atendimentos pré e pós testagem, nas consultas de enfermagem e no próprio aconselhamento, também considerado um agir educativo. Para isso, é necessário que as ações de educação em saúde utilizem metodologias que adotem fontes de informações mais acessíveis, como as mídias sociais e a internet. Além disso, é importante que atuem na desconstrução de crenças e atitudes negativas acerca da doença, promovendo comportamentos positivos em relação às práticas sexuais seguras e saudáveis (Shimizu, 2021). Nesse sentido, os profissionais de saúde, especialmente os de nível superior, possuem um duplo papel de educador, com a população e com a equipe de saúde, incluindo a capacitação de auxiliares e técnicos de enfermagem para uma assistência adequada às pessoas que vivem com HIV, suas famílias e suas demandas complexas (Teixeira \& Oliveira, 2014).

Observou-se que o conjunto das atividades educativas é reconhecido pelo gestor como sendo exercido pelo enfermeiro. Essa atribuição não se coaduna com as recomendações do Ministério da Saúde, nas quais é recomendado que todos os membros da equipe desenvolvam essas atividades, por seu papel fundamental para as estratégias de prevenção (Brasil, 2017c). Nesse particular, deve-se destacar que o quantitativo de profissionais e as oportunidades de contato direto com os clientes são maiores para os auxiliares e técnicos de enfermagem, potencializando as oportunidades de desenvolvimento de ações educativas. Esse achado aponta, ainda, a invisibilidade de ações cotidianas efetivadas por esses profissionais, levando ao não reconhecimento do caráter educativo da maior parte das ações de cuidado de enfermagem.

Outra atividade apontada pelos gestores é a consulta de enfermagem, atividade exclusiva do enfermeiro, segundo a lei do exercício profissional (Brasil, 1986), que permite a qualificação e diferenciação do agir do enfermeiro no âmbito da equipe de enfermagem e da equipe de saúde. Destaca-se um baixo percentual dessa atividade neste estudo, apontando para uma não diferenciação do agir do enfermeiro nos serviços de atenção à PVHIV. Em contrapartida, um estudo realizado apontou que os pacientes reconhecem a atuação do enfermeiro, uma vez que todos os participantes do estudo relataram utilizar o serviço de enfermagem e 97\% avaliaram o nível de satisfação como "bom ou muito bom", indicando a importância do papel do profissional enfermeiro (Guilherme, Yamaguchi \& Massuda, 2019).

No contexto do HIV/aids, uma pesquisa realizada com enfermeiros de um serviço ambulatorial revelou que a escuta ativa durante a realização da consulta de enfermagem se constituiu como a principal estratégia para a construção de uma relação de empatia e confiança com o usuário, viabilizando a manifestação de dúvidas, temores, medos e anseios (Macedo, Sena \& Miranda, 2013). Ainda, a consulta de enfermagem possibilita a disseminação de informações e orientações sobre o HIV e a aids, assim como a convocação do indivíduo para a participação ativa no processo de cuidado, buscando conscientizálo acerca da importância da adesão ao tratamento medicamentoso. A consulta de enfermagem configura-se como mais um momento educativo favorável à troca de saberes e estreitamento de laços (Macedo et al., 2013).

Estudo identificou que a consulta de enfermagem permite a satisfação biopsicossocial dos usuários por meio da atenção dispensada pelo enfermeiro durante a consulta, que resulta em sensação de bem-estar após o atendimento. Essa modalidade de atendimento é percebida como um espaço de acolhimento, pois possibilita o diálogo, a livre expressão de dúvidas, sentimentos e experiências, resultando na construção de vínculo entre enfermeira e paciente (Oliveira et al, 2012).

O cuidado domiciliar foi mencionado pelos gestores, mas com pequena expressão quantitativa e atribuído principalmente aos enfermeiros, da mesma forma que nas demais atividades. Considerando que essa atividade é estruturadora da política de atenção para reorganização dos fluxos de atendimento com descentralização do acompanhamento das PVHIV do SAE para a Estratégia de Saúde da Família, que se encontrava em processo de implementação à época da coleta de dados, seria 
esperado que a visita domiciliar já se apresentasse como ação destacada nos serviços (Soranz, Pinto \& Penna, 2016; Ferreira Neto, Oliveira, Viana \& Duarte, 2016).

No entanto, estudo de Goulart et al (2021) aponta que a visita domiciliar é, prioritariamente, destinada à busca de usuários com alguma limitação de acesso à unidade, restrição ao leito ou domicílio. Situações como incursão policial, confronto armado são limitadores para a realização da visita domiciliar. A qualidade da atenção prestada fica prejudicada e as atividades no território sofrem grandes prejuízos, trazendo medo e insegurança, inclusive aos profissionais. Considera-se que objetivo da visita domiciliar vai muito além da dificuldade ou impossibilidade física de acesso do usuário, e qualquer outra pessoa cadastrada nos serviços, que necessite de cuidados ou não, deverá receber o cuidado domiciliar quando for necessário ou indicado. Além disso, a atividade configura-se como uma oportunidade de construção de vínculo, aproximação entre a equipe e a clientela adscrita, e produção de saúde nos territórios (Kebian \& Acioli, 2014; Alonso, Beguin \& Duarte, 2018)

Nesta análise observou-se uma atribuição discreta da consulta de enfermagem aos técnicos de enfermagem, o que representa um viés de percepção dos gestores, uma vez que essa é uma atividade exclusiva do enfermeiro, conforme citado anteriormente. Uma segunda distorção observada nos resultados foi a atribuição da primeira consulta ao cliente com HIV aos técnicos de enfermagem, contrariando a recomendação do Ministério da Saúde de que essa atividade seja realizada por profissionais de nível superior, como médico, enfermeiro, psicólogo, assistente social ou outros profissionais capacitados que sejam integrantes da equipe multiprofissional (Brasil, 2017c). Finalmente, a atribuição de atendimentos psicológicos a sociais aos enfermeiros também aponta uma percepção distorcida do papel próprio dos membros da equipe de saúde e de enfermagem, uma vez que esses atendimentos são específicos de Assistentes Sociais e Psicólogos. Conforme apontam Gomes et al. (2010), Thiengo et al. (2010) e Formozo \& Oliveira (2010) o enfermeiro parece exercer um papel de amálgama das ações dos demais membros da equipe multiprofissional nos serviços de saúde, o que leva a invisibilidade do seu papel próprio.

Ressalta-se que a assistência multiprofissional e contínua às PVHIV deve englobar ações de promoção da saúde, prevenção e tratamento de doenças, visando garantir um olhar complexo às necessidades desse grupo, que perpassa por dimensões que extrapolam o cuidado físico e a disponibilização de medicamentos antirretrovirais, incluindo acesso digno aos serviços de saúde, com assistência integral, de qualidade e atendimento livre de preconceito (Abrão et al., 2014; Brasil, 2017d).

\section{Conclusão}

Observou-se neste estudo que, dentre as ações reconhecidas pelos gestores nos serviços de atendimento às PVHIV, a maior parte foi associada ao enfermeiro, embora compreenda também as ações dos demais membros da equipe de enfermagem. As atividades destacadas foram aquelas relativas à consulta de enfermagem, à primeira consulta, oferta de exame anti-HIV, ao atendimento domiciliar, além de atividades educativas, como aconselhamento individual e coletivo. As atividades de prevenção e, particularmente, a sua faceta educativa, foram reconhecidas como atributo da equipe de enfermagem, mesmo que focalizadas no enfermeiro. Esse achado demonstra, por um lado, o reconhecimento pelos gestores da importância do enfermeiro na equipe, e por outro, pouca clareza quanto aos diferentes papéis exercidos pelos profissionais da equipe de enfermagem na atenção a esse grupo.

Algumas distorções na percepção dos gestores foram observadas. Elas se referem à atribuição aos enfermeiros dos atendimentos psicológico e social, à consulta de enfermagem atribuída aos técnicos e auxiliares de enfermagem, além da primeira consulta como atividade específica do enfermeiro, o que indica pouca clareza dos gestores sobre a especificidade do trabalho da equipe de enfermagem e do enfermeiro em particular, além da invisibilidade de uma prática cotidiana de cuidado que se revela fundamental para o PN DST/aids.

Considera-se que o processo de trabalho assistencial deve estar baseado na escuta ativa e no acolhimento às PVHIV. Não implica apenas reconhecer a diversidade subjetiva, mas é necessário abrir-se a histórias de vida e à intersubjetividade para 
atuar nessas dimensões. Isto requer a construção de aprendizagens conjuntas mediante a tecnologia básica que é o diálogo. O diálogo pressupõe troca, contato corporal, sensibilização, o lúdico, o ético, mobilizando conhecimentos e práticas mediados pelas dimensões afetivo-emocional, físico-corporal e cognitiva.

A Rede de Atenção à Saúde é estruturada a partir de atenção básica, sendo o primeiro ponto de atenção e principal porta de entrada do sistema de saúde, constituída por equipe multidisciplinar que integra e coordena o cuidado, sendo um de seus componentes a definição de linhas de cuidado. Neste sentido, torna-se primordial a definição de linhas de cuidado voltadas para as PVHIV, como já ocorre em alguns municípios. Assim, o gestor é responsável pelo planejamento e por assegurar o desenvolvimento das atividades essenciais à realização dessas linhas de cuidado às PVHIV, que pode ser comprometida pela falta de clareza das atribuições da equipe de enfermagem.

Como limitação deste estudo convém destacar a opção pela amostra de conveniência e não o cálculo amostral para escolha dos participantes e dos serviços. Para redução dos impactos dessa limitação foi composta uma amostra estratificada por região do país, assegurando alguma representatividade regional e de diferentes contextos do sistema de saúde e do PN DST/aids.

Espera-se que os resultados da presente pesquisa contribuam para a reflexão de gestores e profissionais de saúde sobre o lugar ocupado e as atribuições próprias dos diferentes profissionais participantes da equipe de enfermagem no desenvolvimento da linha de cuidado voltada para as PVHIV, bem como da sua importância para a promoção da qualidade de vida das PVHIV. Sugere-se o desenvolvimento de novos estudos analisando as mudanças ocorridas na atuação da equipe de enfermagem desde a constituição do PN DST/aids, identificando as mudanças ocorridas, não só nas atividades exercidas pela enfermagem, mas, também, no seu reconhecimento profissional frente os demais profissionais e à população usuária dos serviços.

A presente pesquisa recebeu os seguintes financiamentos: CNPq - Recursos financeiros e Bolsa de Produtividade em Pesquisa; UERJ - Bolsas Pro-Ciência e Iniciação Científica; CAPES - Bolsas de Mestrado e Doutorado.

\section{Referências}

Abrão, F. M. S., Angelim, R.C. M., Cardoso, M. D., Queiroz, S. B. A., Freitas, R. M. M., \& Oliveira, D. C. (2014) Características estruturais e organizacionais de serviços de assistência especializada em HIV/aids na cidade de Recife, Brasil. Rev baiana saúde pública; 38(1),140-154.

Alonso, C. M. C., Beguin, P. D., \& Duarte, F. J. C. M. (2018). Trabalho dos agentes comunitários de saúde na Estratégia Saúde da Família: metassíntese. Revista de Saúde Pública, 52(14),1-13.

Brasil. (1986). Lei 7.498, de 25 de junho de 1986. Dispõe sobre a Regulamentação do Exercício da Enfermagem e dá outras providências. Brasília: Ministério da Saúde.

Brasil. Ministério da Saúde (1999a). Coordenação Nacional de DST e aids. Política Nacional de DST/aids: princípios e diretrizes. Brasília

Brasil. Ministério da Saúde (1999b). Coordenação Nacional de DST/ aids. A Resposta Brasileira ao HIV/ aids: Experiências Exemplares. Brasília

Brasil. Ministério da Saúde. (2008a). Secretaria de Vigilância em Saúde. Programa Nacional de DST e Aids. Contribuição dos centros de testagem e aconselhamento para universalizar o diagnóstico e garantir a equidade no acesso aos serviços. Brasília.

Brasil. Ministério da Saúde (2008b). Secretaria de Vigilância em Saúde. Programa Nacional de DST e Aids. Manual de prevenção das DST/HIV/AIDS em comunidades populares. Brasília.

Brasil. Ministério da Saúde. (2013). Portaria Conjunta número 1 de 16 de janeiro de 2013. Altera na Tabela de Serviço Especializado no Sistema de Cadastro Nacional de Estabelecimentos de Saúde (SCNES), o Serviço 106 - Serviço de Atenção a DST/HIV/Aids, e institui o Regulamento de Serviços de Atenção às DST/HIV/Aids, que define suas modalidades, classificação, organização das estruturas e o funcionamento. Brasília.

Brasil. Ministério da Saúde. (2017a). Secretaria de Vigilância em Saúde. Departamento de DST, Aids e Hepatites Virais. Caderno de Boas Práticas em HIV/aids na Atenção Básica. Brasília. http://www.aids.gov.br/pt-br/pub/2014/caderno-de-boas-praticas-em-hivaids-na-atencao-basica.

Brasil. Ministério da Saúde. (2017b). Secretaria de Vigilância em Saúde. Departamento de Vigilância, Prevenção e Controle das Infecções Sexualmente Transmissíveis, do HIV/Aids e das Hepatites Virais. Boletim Epidemiológico HIV Aids 2017. Brasília. 
Brasil. Ministério da Saúde. (2017c). Secretaria de Vigilância em Saúde. Departamento de Vigilância, Prevenção e Controle das Infecções Sexualmente Transmissíveis, do HIV/Aids e das Hepatites Virais. Diretrizes para organização do CTA no âmbito da Prevenção Combinada e nas Redes de Atenção à Saúde. Brasília: Ministério da Saúde.

Brasil. Ministério da Saúde. (2017d). Protocolo Clínico e Diretrizes Terapêuticas para Manejo da infecção pelo HIV em adultos. Brasília.

Brasil. Ministério da Saúde. (2018). Secretaria de Vigilância em Saúde. Departamento de Vigilância, Prevenção e Controle das Infecções Sexualmente Transmissíveis, do HIV/Aids e das Hepatites Virais. Manual técnico para o diagnóstico da infecção pelo HIV em adultos e crianças. Brasília.

Brasil. Ministério da Saúde. (2020, dez) Secretaria de Vigilância em Saúde. Boletim Epidemiológico HIV/aids, número especial, dez 2020.

Centro de Referência e Treinamento em DST/Aids, Coordenação do Programa Estadual DST/Aids-SP, Coordenadoria de Controle de Doenças, Secretaria de Estado da Saúde de São Paulo. (2009). Recomendações para o funcionamento dos Centros de Testagem e Aconselhamento (CTA) do estado de São Paulo. Rev Saúde Pública, 43(2), 383-386.

Ferreira Neto, J. L., Oliveira, G. L, Viana, N. O, \& Duarte, L. G. M. F (2016). Integralidade, condições de oferta de serviços e processo de trabalho de Equipes de Saúde da Família em Belo Horizonte. Saúde Debate, 40(111),179-92.

Formozo, G. A., \& Oliveira, D. C. (2010) Representações sociais do cuidado prestado aos pacientes soropositivos ao HIV. Rev Bras Enferm. 2010; 6(3), 2307.

Gomes, A. M. T, Thiengo, P. C. S., Anunciação, C. T, Oliveira, D. C, \& Kestenberg, C. C. F. (2011). Representações sociais das atividades da enfermagem junto aos pacientes soropositivos: caracterizando ações e atores sociais. Rev Eletr Enf, 13(1):16-23.

Goulart E. P., Moura, A. T. M. S., Rafael, R. M. R., Edmundo, K. M. B., \& Penna, L. H. G. (2021). Visita domiciliar pela Estratégia Saúde da Família: limites e possibilidades no contexto da violência urbana no Rio de Janeiro. Rev Bras Med Fam Comunidade, 16(43), 2651

Guilherme, J. A., Yamaguchi, M. U. \& Massuda, E. M. (2019). Satisfação dos portadores de HIV/AIDS com o serviço de assistência especializada. Revista Mineira de Enfermagem, 23(e-1213).

Kebian, L. V. A, \& Acioli, S. (2014). A visita domiciliar de enfermeiros e agentes comunitários de saúde da Estratégia Saúde da Família. Revista Eletrônica de Enfermagem, 16(1),161-9.

Macedo, S. M; Sena, M. C. S \& Miranda, K. C. L. (2013). Consulta de enfermagem ao paciente com HIV: perspectivas e desafios sob a ótica de enfermeiros. Rev Bras Enferm; 66(2), 196-201.

Nemes, M. I. B., Caraciolo, J. M. M., Santos, M. A., Alves, A. M., Yokaichiya, C. M., Prado, R. R, Vale, F. C, \& Basso, C. R. (2011). Avaliação da qualidade dos serviços ambulatoriais do SUS que assistem adultos vivendo com HIV/Aids no Brasil. (Relatório da Aplicação de 2010 e Comparação com o resultado de 2007). Departamento de DST, Aids e Hepatites Virais, Secretaria de Vigilância em Saúde, Ministério da Saúde. São Paulo.

Oliveira, D. C., Gomes, A. M. T., Marques, S. C., Penna, L. H. G., Sá, C. P., Wolter, R. M. C. P, \& Strasburg, A. (2009). As Transformações do Cuidado de Saúde e Enfermagem em Tempos de AIDS: estrutura ambulatorial, representações sociais e memórias de enfermeiros e profissionais de saúde no Brasil. Projeto de Pesquisa. Rio de Janeiro.

Oliveira, S. K. P., Queiroz, A. P. O., Matos, D. P. M., Moura, A.F., \& Lima, F. E. T., (2012). Temas abordados na consulta de enfermagem: revisão integrativa da literatura. Rev. Bras. Enferm., 65(1), 155-161.

Pereira, F. W., Fonseca, A. D., Oliveira, D. C., Fernandes, G. F. M \& Marques, S. C. (2015). Transformação das práticas profissionais de cuidado diante da AIDS: representações sociais dos profissionais de saúde. Rev enferm UERJ, 23(4), 455-460.

Ribeiro, E.D.S., Cavalcante, L. P. A., Durans, K. C. N., Leite, A. M., Miranda, A. M. C., Barbosa, E. M. R., Sousa, H. F., \& Batista, M. C. A. (2021) Diagnósticos e intervenções de Enfermagem acerca da qualidade de vida da pessoa que vive com HIV: revisão integrativa. Research, Society and Development, 10(10). https://rsdjournal.org/index.php/rsd/article/view/18935/16852

Shimizu, H. E. (2021) Representações sociais acerca do HIV/AIDS e a gestão de riscos em tempos de cronificação da doença. Research, Society and Development, 10(10). e257101018357.https://rsdjournal.org/index.php/rsd/article/view/18357/16800

Soranz, D., Pinto, L. F, \& Penna, G. O. (2016). Eixos e a Reforma dos Cuidados em Atenção Primária em Saúde (RCAPS) na cidade do Rio de Janeiro, Brasil. Ciência \& Saúde Coletiva, 21(5), 1327-38.

Teixeira, E \& Oliveira, D. C. (2014) Representações sociais de educação em saúde em tempos de AIDS. Rev Bras Enferm, 67(5), 810-817.

Villarinho, M. V., Padilha, M. I., Berardinelli, L. M. M., Borenstein, M. S., Meirelles, B. H. \& SAndrade, S. R. (2013). Políticas públicas de saúde face a epidemia da aids e a assistência as pessoas com a doença. Rev Bras Enferm; 66(2), 271-277. 